\section{Life-threatening hypokalemia associated with profound electrocardiographic manifestations in thyrotoxic periodic paralysis}

\author{
Priyanka Lall, Razvan Dadu, \\ Anabelle Morales, Stuart Zarich \\ Department of Medicine and Division \\ of Cardiovascular Medicine, Bridgeport \\ Hospital, Bridgeport Ct. Yale University \\ School of Medicine, USA
}

\section{Abstract}

We describe a case of thyrotoxic periodic paralysis (TPP) associated with profound electrocardiographic abnormalities and cardiac arrhythmias along with a brief literature review. A 38-year old Hispanic male presented with a history of intermittent profound lower extremity weakness which went undiagnosed for over a year before a diagnosis of thyrotoxic periodic paralysis (TPP) was made. The patient experienced ventricular tachycardia, as well as complete atrioventricular block and a right bundle branch block requiring urgent treatment. Although all these electrocardiographic abnormalities are known to occur in TPP, they are rarely manifest together in a single patient. TPP is a frequently unrecognized but life-threatening disorder which requires a high index of suspicion for prompt diagnosis and treatment. Our patient demonstrates the multiple electrocardiographic abnormalities associated with TPP and severe hypokalemia. Unfortunately, this condition went unrecognized for over a year, highlighting the need for better recognition of this potentially fatal disorder.

\section{Introduction}

Thyrotoxic periodic paralysis (TPP) is a rare complication of hyperthyroidism which occurs most frequently in Asian men. TPP commonly precedes the overt symptoms of hyperthyroidism and may be associated with a range of different electocardiographic (ECG) abnormalities. Ventricular dysrhythmias during the paralytic attack are uncommon and have been described in only sporadic case reports. Additionally, although PR prolongation is common, high degree AV block is rarely seen. We describe a 38-year old Hispanic male with TPP who presented with intermittent episodes of profound weakness and difficulty walking which went undiagnosed over the course of more than a year. This profile was associated with profound ECG abnormalities, including both $3^{\text {rd }}$ degree heart block and a potentially fatal ventricular arrhythmia. We present a brief review of the literature of TPP to outline the clinical presentation, pathophysiology, ECG abnormalities and treatment of this potentially fatal condition which requires prompt diagnosis and therapy. A PubMed search from 1960 to the present was conducted using the search terms TPP, periodic paralysis, hypokalemia and thyrotoxicosis.

\section{Case Report}

A previously healthy 38-year old Hispanic male presented to the hospital with difficulty walking and generalized muscle weakness. A little over one year earlier he had presented to the emergency department with a similar episode of lower extremity weakness. He had been evaluated with $X$ rays of the pelvis and sent home with reassurance. No laboratory tests were performed at that time. The symptoms initially resolved spontaneously.

The patient's symptoms reoccurred on an intermittent basis throughout the following year with more pronounced weakness in his lower extremities compared to his upper extremities which did not improve with rest. He had no significant past medical history or prior medication use. He denied any recent infection, exposure to cold or excessive emotional stress. He denied the use of diuretics, laxatives, alcohol or recreational drugs. He reported associated intermittent palpitations, night sweats, nausea and vomiting and diarrhea (6-7 bowel movements/day). He complained of a reduced appetite with a 20 pound weight loss. The patient admitted to drinking up to 3 gallons of water daily. His family history was significant for hyperthyroidism, as well as thyroid carcinoma in his mother.

On physical examination, he had a mildly enlarged non-tender thyroid with a firm consistency with an associated bruit. There were no nodules present. A grade 3/6 holosystolic murmur was recorded throughout the precordium with radiation to both the axilla and neck with a hyperdynamic precordium. The patient had normal muscle tone and no signs of rigidity. There was no muscle atrophy. Muscle strength was $1 / 5$ in the lower extremities bilaterally and $4 / 5$ in the upper extremities Tendon reflexes were absent in both the upper and lower extremities. Proximal weakness was more defined compared to distal weakness. He had no exopthalmos, or sensory or cranial nerve deficits.

On admission, serum potassium was 1.7 $\mathrm{mEq} / \mathrm{L}$, with serum phosphorus $2.5 \mathrm{mg} / \mathrm{dL}$ and
Correspondence: Priyanka Lall, Bridgeport Hospital, 267 Grant St, Bridgeport, CT 06610, USA. Tel. +1.585.309.6907 - Fax: +1.203.330.7497.

E-mail: lallpriyanka@hotmail.com

Conflict of interest: the authors report no conflicts of interest.

Received for publication: 21 January 2011.

Revision received: 1 July 2011.

Accepted for publication: 20 October 2011.

This work is licensed under a Creative Commons Attribution NonCommercial 3.0 License (CC BYNC 3.0).

(C) Copyright P. Lall et al., 2011

Licensee PAGEPress, Italy

Endocrinology Studies 2011; 1:e13

doi:10.4081/es.2011.e13

magnesium $1.3 \mathrm{mg} / \mathrm{dL}$. The rest of the electrolytes and renal function were within normal limits. Urine and serum toxicology were negative. Creatinine phosphokinase levels and liver function were within normal limits. Troponin I levels were abnormal $(0.14 \mathrm{ng} / \mathrm{mL}$ initially and $0.67 \mathrm{ng} / \mathrm{mL}$ on serial testing). A random cortisol level was normal at $25 \mathrm{mcg} / \mathrm{dL}$. The initial ECG showed sinus tachycardia with Mobitz type I $2^{\text {nd }}$ degree A-V Block with an associated non-specific intraventricular conduction delay and a prolonged corrected QT interval. Twenty minutes later the patient went into transient complete heart block with a right bundle branch escape rhythm.

The thyroid stimulating hormone level subsequently returned to less than $0.015 \mathrm{mIU} / \mathrm{mL}$ with a free thyroxine (T4) level of $5.88 \mathrm{ng} / \mathrm{dL}$ and a T3 total of more than $800 \mathrm{ng} / \mathrm{dl}$, confirming a diagnosis of thyrotoxicosis. An anti-thyroid peroxidase antibody level of 827 units/mL, along with an anti- thyroglobulin level of 572 units/mL confirmed the diagnosis of Graves' disease. ANA titer was less than 1:40. The patient was given $40 \mathrm{mEq}$ of IV potassium replacement at $10 \mathrm{e} / \mathrm{h}$ and started on a normal saline infusion with $20 \mathrm{mEq} / \mathrm{l}$ of potassium and IV magnesium replacement. Subsequently, the patient experienced an episode of sustained ventricular tachycardia requiring a lidocaine bolus and subsequent infusion for conversion to sinus rhythm. He was started on methimazole $20 \mathrm{mg}$ per day and switched to an oral potassium replacement protocol, which resulted in the complete resolution of his lowerextremity paralysis. On the third day of admis sion, potassium was $5.1 \mathrm{mEq} / \mathrm{L}$, phosphorus 4.1 $\mathrm{mg} / \mathrm{dL}$, and magnesium $1.8 \mathrm{mg} / \mathrm{dL}$. Serial measurements of serum potassium level in the hospital remained within normal limits and his ECG normalized. The patient was discharged home with the diagnosis of TPP sec- 
ondary to Graves' disease. A thyroid uptake scan was scheduled on an outpatient basis.

\section{Discussion}

Acute muscle weakness is a non-specific symptom associated with a variety of benign to life-threatening disorders. Among the metabolic causes, TPP is an alarming and potentially lethal complication of hyperthyroidism characterized by muscle paralysis and hypokalemia due to a massive shift of potassium to the intracellular space. Morbidity and mortality in patients with unrecognized TPP are typically related to hypokalemic complications, such as ventricular arrhythmia and respiratory failure.

TPP is a well-known complication of thyrotoxicosis in Asian populations, but is relatively uncommon in other ethnicities. The reported overall incidence of TPP in China and Japan is approximately $2 \%{ }^{1,2}$ In the United States, the incidence of TPP in non-Asian populations is approximately one tenth of that found in Asian countries $^{3}$ and is thus frequently underappreciated by clinicians. TPP is not infrequently misdiagnosed in Western countries because of its similarities to familial periodic paralysis (FPP). Biochemical hyperthyroidism distinguishes patients with TPP from FPP. TPP may follow treatment with steroid treatment initiated to prevent the ocular symptoms in Graves' disease. $^{4}$

While thyrotoxicosis commonly affects women, TPP is predominantly seen in men in the second and fourth decades of life. ${ }^{4,5}$ Patients present with recurrent transient episodes of muscle weakness that range from mild weakness to complete flaccid paralysis. Attacks usually involve proximal symmetrical ascending lower-extremity muscle weakness during the early morning hours. Acute episodes may be preceded by muscle aches, cramps, and muscle stiffness. Other precipitating factors include trauma, exposure to cold, emotional stress, infection, alcohol and drugs.

Deep tendon reflexes are generally absent. Total paralysis of respiratory, bulbar and ocular muscles has been reported in severe attacks..$^{6-8}$ The paralysis can last 3-96 $\mathrm{h}$ and typically resolves in the reverse order from which events occurred. Findings of thyrotoxicosis, such as warm and moist skin, fever, tachycardia, exopthalmos, or goiter may be subtle but are important clues to the diagnosis. The majority of cases of hyperthyroidism associated with TPP are due to Graves' disease, although other conditions including thyroiditis, toxic nodular goiter, and toxic adenoma have also been implicated.

The pathogenesis of hypokalemic periodic paralysis in certain populations with thyrotoxicosis is unclear. Thyroid hormone coupled
A

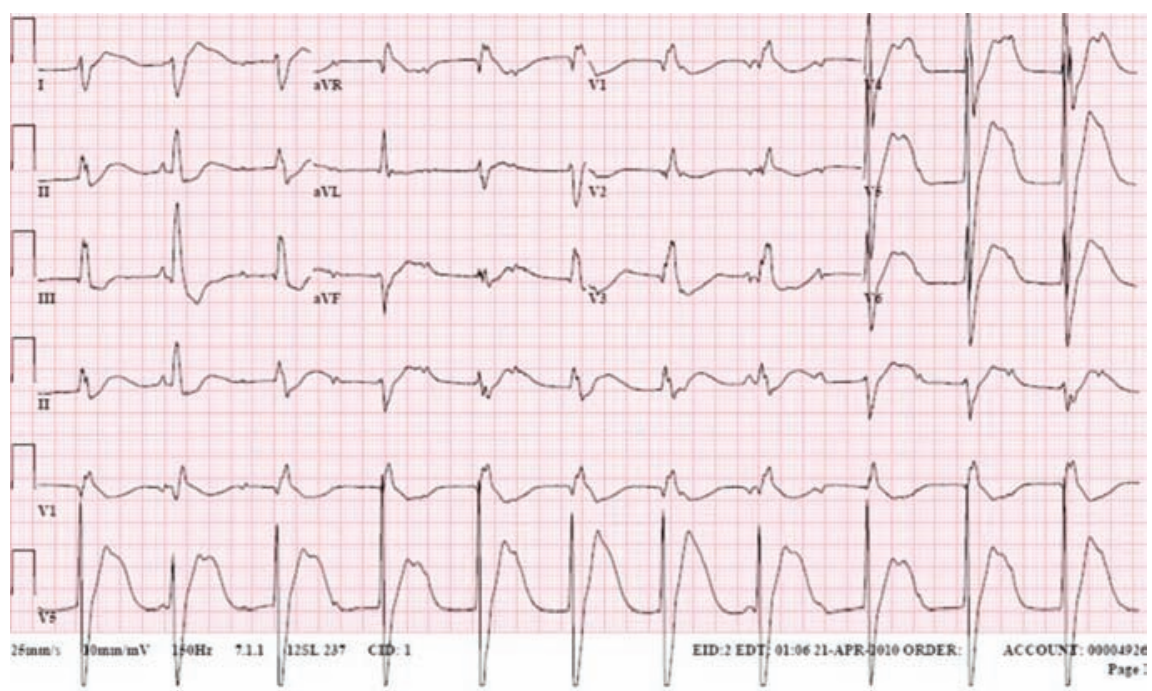

B
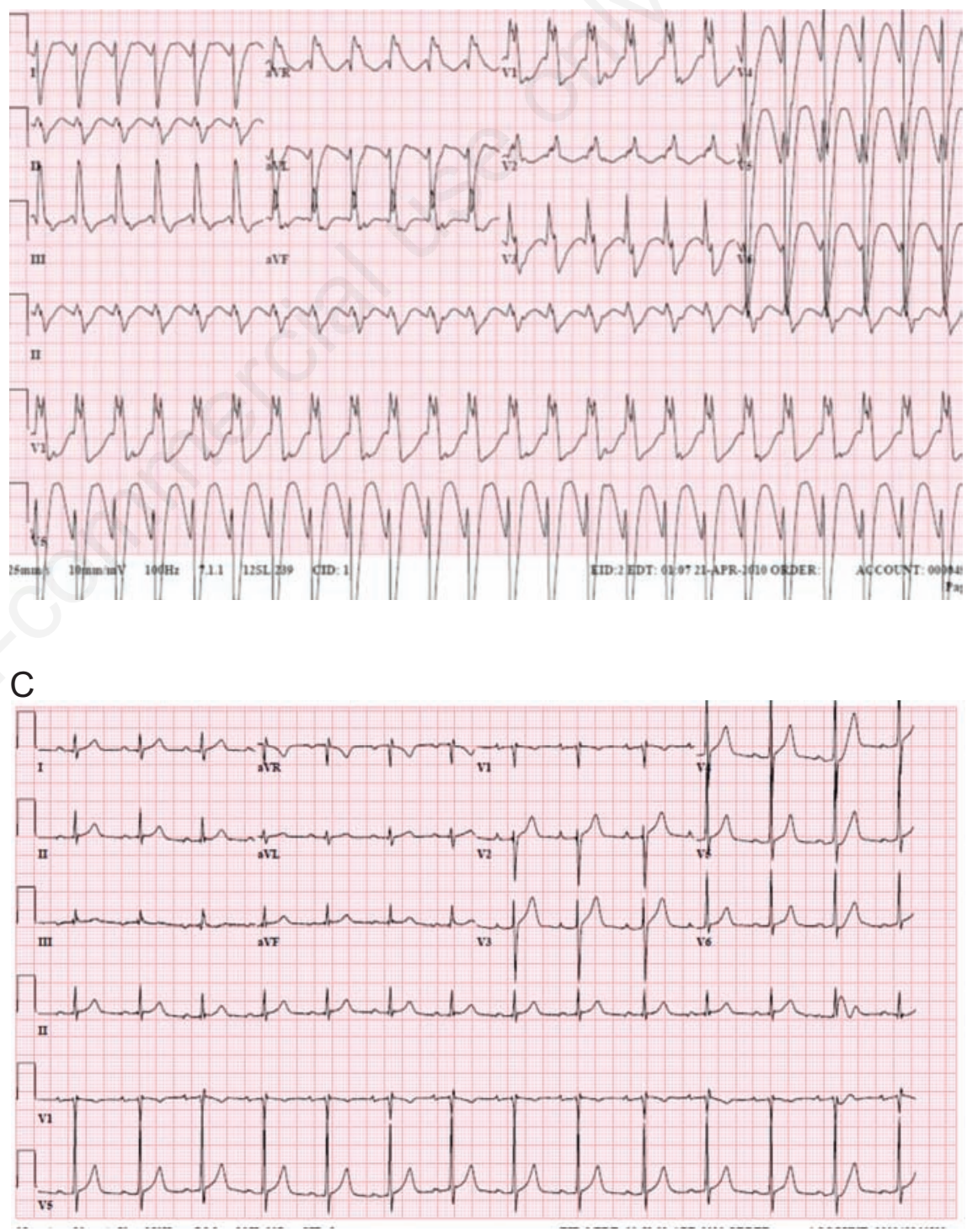

Figure 1. (A) Presenting electrocardiogram with complete heart block, right bundle branch, markedly prolonged QT interval and pseudo-myocardial infarction pattern in lateral leads. (B) Ventricular Tachycardia with 2:1 retrograde AV conduction. (C) Normal electrocardiogram after treatment with normalized serum potassium levels. 
with beta adrenergic hyperactivity in a thyrotoxicosis state increases Na/K-ATPase activity, leading to influx of potassium into the intracellular space causing extracellular hypokalemia without a deficit in total body potassium stores. Other causes of paralysis in the setting of hypokalemia are typically due to excessive total body potassium deficits. Chan et al. found an increase in platelet Na/K-ATPase activity in TPP patients which was not present in thyrotoxic patients without paralysis. ${ }^{9}$ Additionally, an exaggerated response of $\mathrm{Na} / \mathrm{K}$-ATPase activity to adrenaline has also been noted in TPP patients. ${ }^{10}$ Hyperthyroidism also increases the number and sensitivity of Na/K-ATPase pumps, as well as beta adrenergic sensitivity. ${ }^{11}$ In addition to hypokalemia, there may be hypophosphatemia due primarily to a shift of phosphate into cells during an attack. Hypomagnesemia may also be present due to a similar intracellular shift, likely secondary to endogenous catecholamine released during stress rather than due to depletion of body stores. These electrolyte disturbances typically correct as the acute thyrotoxicosis resolves.

Electrocardiographic findings may be characteristic of hypokalemia, with increased Pwave amplitude, ST segment depression, prominent $\mathrm{U}$ waves, prolonged PR and QT intervals, and widened QRS complexes. Attacks can also be associated with higher degree AV block, intraventricular conduction delays and ventricular arrhythmias, as well as asystole; 12 essentially all of which, with the exclusion of asystole, were manifest in our patient (Figures 1 A-C). Ventricular arrhythmias are rare, however, during paralytic attacks, only being seen in sporadic case reports. ${ }^{13}$ In fact, in a review of 31 patients with TPP, no patients experienced ventricular tachycardia despite profound hypokalemia (mean $2.0 \pm 0.6 \mathrm{mmol} / \mathrm{L}$ ). ${ }^{14}$ Additionally, in that study, $45 \%$ of patients had a prolonged PR interval, but only $10 \%$ had higher degrees of $\mathrm{AV}$ block. Elevated QRS voltage, which is commonly seen in hyperthyroidism independent of hypokalemia, was the most common finding in that study (74\%) and was also noted in our patient.

During periodic paralysis and marked hypokalemia, immediate supplementation with potassium chloride is warranted to prevent major cardiopulmonary and respiratory complications. However, as body stores of potassium are normal in TPP, caution is warranted, as up to $40 \%$ of patients given intra- venous $\mathrm{KCl}$ may develop rebound hyerkalemia. ${ }^{5}$ Oral or IV propranolol, a nonspecific B-adrenergic blocker has also been proposed as an alternative treatment to ameliorate the paralysis while avoiding rebound hyperkalemia and raising serum levels of potassium and phosphate. ${ }^{15}$ However, in view of the small number of case reports on the use of B-blockers alone, more controlled studies are needed to determine their efficacy compared with potassium supplementation in the emergency treatment of TPP. Potassium should be infused with saline as a glucose infusion may worsen hypokalemia by shifting potassium to the intracellular space. ${ }^{16}$

\section{Conclusions}

Our patient highlights the fact that TPP, although uncommon in Western populations, is a rare disease associated with thyrotoxicosis which requires prompt recognition and treatment to avoid potentially life-threatening complications. The pathophysiology of TPP appears to be secondary to a shift in potassium to the intracellular space rather than excess potassium losses and may lead to fatal cardiac arrhythmias. Electrocardiographic abnormalities are related to the degree of hypokalemia and may result in both life-threatening heart block and ventricular arrhythmias. Our patient manifested both of these electrocardiac findings which were indeed life-threatening in his case. It is recommended that potassium should be replaced with caution to prevent rebound hyperkalemia which can further lead to fatal arrhythmias. Our case highlights the difficulty of making an early diagnosis if there is an inadequate index of suspicion.

\section{References}

1. McFadzean AJS, Yeung R. Periodic Paralysis complication thyrotoxicosis in Chinese. Br Med Journal 1967;1:451-5.

2. Okinaka S, Shizume K, Lino S, Watanabe $\mathrm{A}$, Irie $\mathrm{M}$ et al. The association of periodic paralysis and hyperthyroidism in Japan. $\mathrm{J}$ Clin Endocrinol Metab 1957;17:1454-9.

3. Kelley DE, Gharib H, Kennedy FP. Thyrotoxic Periodic Paralysis: Report of 10 cases and review of electromyographic findings. Arch Intern Med 1989;149:2597600.

4. Wongraoprasert $S$, Buranasupkajorn $P$, Sridama V, Snabboon T. Thyrotoxic periodic paralysis induced by pulse methylprednisolone. Intern Med 2007;46:1431-3.

5. Manoukian MA, Foote JA, Crapo LM. Clinical and metabolic features of thyrotoxic periodic paralysis in 24 episodes. Arch Intern Med 1999;159:601-6.

6. Liu YC, Tsai WS, Chau T, Lin SH. Acute hypercapneic respiratory failure due to thryotoxic periodic paralysis. AM J Med Sci 2004;327:264-7.

7. Ahlawat SK, Sachdev A. Hypokalemic paralysis.Postgrad Med J 1999;75:193-7.

8. Crane MH, 1960 Periodic Paralysis associated with hyperthyroidism. Calif Med 1960: 92;285-8.

9. Chan A, Shinde R, Chow C, Cockram C, Swaminathan R. Hyperinsulinemia and $\mathrm{Na}+, \mathrm{K}+$ ATPase activity in subjects with thyrotoxic periodic paralysis. Clin Endo 1994;41:213-6.

10. Chronos N, Mendel P, Ozin R. Thyrotoxicosis presenting as a life threatening hypokalemic paralysis:investigation of the $\mathrm{Na} / \mathrm{K}$ pump in isolated leukocytes. $\mathrm{Br} \mathrm{J}$ Clin Path 1993;47:106-8.

11. Nora N, Berns A. Hypokalemic, hypophosphatemic thyrotoxiccotic periodic paralysis. Am J Kid Dis 1989;13:247-9.

12. Chung A. Clinical review: Thyrotoxic periodic paralysis: a diagnostic challenge. The Journal of Clinical Endocrinology \& Metabolism 2006;91:2490-5.

13. Loh K, Pinheiro L, Ng K. Thyrotoxic periodic paralysis complicated by near fatal ventricular arrhythmias. Singapore J Med 2005;46:88-9.

14. Hsu Y, Yuh-Feng L, Tom C, Jun-ting L, ShiWen K, Shih-Hua L. Electrocardiographic manifestations in patients with thyrotoxic periodic paralysis. Am J Med Sci 2003;326: 128-32.

15. Kunin A, Surawicz B, Sims E. Decrease in serum potassium level and the appearance of cardiac arrhythmia during infusion of potassium with glucose in potassium depleted patients. N Engl J Med 1962;266: 288-96.

16. Lin S, Lin Y. Propranolol rapidly reverses paralysis, hypokalemia and hypophosphatemia in thyrotoxic periodic paralysis. Am J Kidney Dis 2001;37:3:620-3. 